\title{
Efficacy of a Breastfeeding Support Education Program for Late Preterm Infants: a Randomized Controlled Study
}

Izumi Sato ( $\square$ i-sato@redcross.ac.jp )

Japanese Red Cross College of Nursing https://orcid.org/0000-0002-4371-1617

Masumi Imura

Japanese Red Cross College of Nursing

\section{Research}

Keywords: Breastfeeding, Education Program, Late Preterm Infants, RCT

Posted Date: May 17th, 2021

DOl: https://doi.org/10.21203/rs.3.rs-520210/v1

License: (c) (i) This work is licensed under a Creative Commons Attribution 4.0 International License.

Read Full License 


\section{Abstract}

Background: Nutritional support affects the growth and development of late preterm infants (LPIs) and also impacts their health in the long term. Though breastmilk is the first choice as a nutrition source for LPIs, there is no consensus on how to provide supplements when breastmilk is unavailable, as well as the quantity to be provided or nutritional strategies to be followed. LPIs may need to wait for a certain period until breastmilk supply is sufficient. Nurses and midwives play an important role in supporting the promotion of breastmilk secretion in the postnatal period. Therefore, this study aimed to develop an educational program for them and test its efficacy.

Methods: In this two-group parallel randomized controlled trial, the data were collected between July and April 2019 from nurses and midwives working in obstetrics facilities who were randomly assigned to either the breastfeeding support for LPIs (BSLPI) program or the non-technical skills (NTS) program. The effects of the intervention were assessed immediately before, after, and one month after the program.

Results: There were 36 participants in the BSLPI group and 33 in the NTS group. The BSLPI group had significantly higher self-efficacy for providing breastfeeding support immediately after (mean=55.7, $S D=8.0, p=.001$ ) and one month after the intervention (mean=57.3, $S D=8.6, p=.001$ ) compared to immediately before the intervention (mean $=47.8, S D=10.3$ ). The BSLPI group demonstrated significant improvements in social skills in nursing interactions with mothers immediately after the intervention (BSLPI: mean=80.5, $S D=110.9$ vs. NTC: mean $=75.0, S D=9.7, p=.032$ ) and one month later (BSLPI: mean=82.5, $S D=10.1$ vs. NTC: mean=75.8, $S D=9.4, p=.010$ ), as well as in knowledge and skills necessary for breastfeeding support for LPIs immediately after the intervention (BSLPI: mean $=84.5, S D=8.3$ vs. NTC: mean=48.7, $S D=11.9, p=.001$ ) and one month later (BSLPI: mean=79.3, $S D=11.3$ vs. NTC: mean=51.3, $\mathrm{SD}=12.6, \mathrm{p}=.001)$.

Conclusions: The BSLPI intervention increased nurses' and midwives' sense of self-efficacy for providing breastfeeding support, social skills in nursing interactions with mothers, and knowledge and skills necessary for breastfeeding support for LPIs, suggesting that it provided effective support.

\section{This trial was retrospectively registered with the University Hospital Medical Information Network (UMIN; R000040145UMIN000035227).}

\section{Background}

Infants born between 34 weeks, 0 days, and 36 weeks, 6 days of gestation are called late preterm infants (LPIs). LPIs account for approximately $80 \%$ of all early-term births [1]. It is a fact that nutritional support in infancy influences future health status, and breastmilk intake is expected to improve the body composition of LPIs to an optimal state, positively influence future health maintenance [2], and potentially optimize gut flora [3]. A recent study reported a trade-off of rapid weight gain in the immediate postnatal period: while it may prevent cognitive delay in LPIs, it also increases the risk of future obesity [4]. 
Breastfeeding is promoted for all neonates [5], and LPIs are no exception. Though breastmilk is the optimal form of nutrition, adhering to the ten steps to successful breastfeeding [5] alone may not help mothers cope with the challenges that arise after hospital discharge [6, 7]. Compared to term infants, LPIs may need to wait longer for the mother to secrete breastmilk. Therefore, LPIs' nutrition may need to be temporarily supplemented with sources other than breastmilk. However, methods of feeding LPIs are different in various facilities, and this is understood to be linked to the lack of guidelines for LPI nutritional support [8]. Thus, a discussion on LPI feeding warrants careful examination and implementation of measures that consider their current needs and ensure their future health. Although it is important for LPIs to be temporarily supplemented with nutritional support through substitutes other than breast milk, it is far more important to lay the foundation for mothers to be able to breastfeed their LPIs. Nurses and midwives, therefore, play an important role in early LPI transition from non-breastmilk to breastmilk nutrition.

A survey on LPIs capable of oral feeding and did not require medical intervention demonstrated that they were significantly less likely to be breastfed than term infants [9-11]. In other studies, mothers of LPIs were devoted to breastfeeding from the postnatal period but experienced stress owing to LPIs not waking up at feeding time, ineffective suckling, hospital policies, and unsatisfactory interactions with their physicians, nurses, and midwives $[9,12,13]$.

Regarding LPI feeding methods in hospitals, $10 \%$ dextrose and infant formula are some foods reportedly administered from the early postnatal period for LPIs capable of oral feeding [14]. Although further investigations are needed, prevention of LPI hypoglycemia, poor weight gain, and ensuring that mothers and their babies can be discharged from the hospital as scheduled are some factors that might explain these practices. However, Allistair reported that overfeeding LPIs with supplementary sources such as dextrose and formula can potentially affect latching and suckling as well as lower breastmilk supply [15]. We, therefore, considered the necessity of implementing medical initiatives to prevent obstacles to breastfeeding.

A previous study demonstrated the benefits of educational interventions in improving knowledge and attitudes among medical staff who provide care for LPIs [16]. Another study reported that having nurses, midwives, and physicians watch a video that demonstrates the necessary skills for breastfeeding support improved their knowledge, acquisition of skills, and self-efficacy [17]. Existing educational programs for nurses and midwives who provide breastfeeding support for LPIs focus on general management, covering only the basics [16]. Moreover, they are limited to techniques and do not include post-discharge breastfeeding support [17]. Knowledge and techniques, as well as communication skills to build good relationships with mothers, are necessary for effective breastfeeding support for LPIs, warranting updates and improvements to the currently available programs. Therefore, the purpose of this study was to conduct an educational program for nurses and midwives who provide breastfeeding support for LPIs and evaluate its efficacy.

\section{Methods}




\section{Design}

A two-group parallel randomized controlled trial, with participants assigned to either the breastfeeding support for LPIs (BSLPI) group or the non-technical skills (NTS) program, was conducted. The effects of the intervention were assessed immediately before, after, and one month after the program.

\section{Sample}

The inclusion criteria were as follows: 1 ) individuals with nursing or midwifery certification working in hospitals, clinics, or midwifery centers that handle deliveries in the Tokyo metropolitan area, 2) two or more years of clinical experience in obstetrics, and 3) experience involving breastfeeding support for LPIs. The exclusion criteria were individuals: 1 ) who were not involved in breastfeeding support for LPIs starting in the early postnatal period because they were not involved in deliveries and 2) in managerial positions, such as head nurses, who were not directly involved in breastfeeding support for LPIs.

\section{Randomization}

Each group was assigned an equal number of nurses and midwives. Additionally, the average duration of experience in the maternity ward was the same for the assigned nurses and midwives. The participants were assigned identification numbers to preserve anonymity. A computerized number generator was used to achieve stratified block randomization [18], and the participants were automatically assigned to the two groups according to this randomization table. Tables and rosters were stored in a locked cabinet and were not accessed until randomization was completed. After randomization, participants were informed of which program they were assigned to in writing, making the study single-blinded.

\section{Sample size calculation}

Statistical power analysis software G*Power [19] was used to calculate the sample size. The necessary sample size was $\mathrm{N}=54$ for an effect size $=0.25$, significance level $=95 \%$, and power $=0.80$ as calculated by repeated measures ANOVA (inter-group and time factors). Assuming a $40 \%$ dropout rate, we aimed to recruit a total of 76 participants (38 in each group).

\section{Intervention}

The educational program was based on the most recent literature on breastfeeding support for LPIs. Specialists in maternity nursing, pediatrics, and breastfeeding support were also consulted to increase content validity. The developed BSLPI program was tested on 18 nurses and midwives to determine its feasibility. The BSLPI intervention significantly increased self-efficacy related to breastfeeding support, social skills in interacting with mothers in nursing settings, and knowledge and skills necessary for breastfeeding support for mothers of LPIs immediately after the intervention, and the improvement was retained one month later.

The content of the BSLPI program can be broadly divided into two segments. The first seeks to increase participants' understanding of LPIs and their mothers' characteristics. The second examines the 
breastfeeding interventions that participants deemed effective for mothers of LPIs in their facilities, such as continual skin-to-skin contact, instructions regarding initial feedings and pumping [20]. These were taught through group work, simulations, and social skills training methods. The intervention itself lasted five hours and was administered through workshops.

The NTS education encompassed non-technical skills that could complement technical know-how [21]. Crew resource management programs, developed to address team and leadership aspects of piloting modern airplanes [22], were also utilized. Such skills and techniques play an important role in medicine and nursing [23-25]. The NTS group was not provided with any information on breastfeeding. The NTS education lasted for five hours and was in the form of a lecture (Table 1).

Table 1

Implementation method and contents of each group program

Breastfeeding support education
program for LPIs
Lecture and group work:
1. Physical characteristics and sucking problems of LPIs
2. Characteristics and lactation problems of mothers with LPIs
3. Points and basis necessary for breastfeeding support for LPI mothers, from
mid-pregnancy till post-discharge: How to do the first feeding; How to use
pump; Skin-to-skin contact

Simulation:

Responding to LPIs during weight loss

Social skills training:

Working with mothers with LPIs who are reluctant to breastfeed

\section{Non-technical skills}

program

\section{Lecture:}

What are nontechnical skills?

\section{Lecture:}

Non-technical skills in the medical field

\section{Lecture:}

Use of nontechnical skills in medical practice

LPIs: late preterm infants

The program was conducted in a small conference room in a convention center located in the Tokyo metropolitan area for both groups.

\section{Assessment}

\section{Self-efficacy related to breastfeeding support}


The Self-Efficacy of Breastfeeding Support (SBS) scale was developed by Toyama et al. based on responses from 729 public health nurses with 5 to 15 years of experience [26]. This scale serves as an indicator of the quality and quantity of potential services, with self-efficacy related to breastfeeding support as a predictor of breastfeeding support behaviors, and this 14-item tool was deemed reliable (Cronbach's $a=0.89$ ). Responses are provided on a five-point scale ( $1=$ not confident at all; $5=$ extremely confident). The total score ranges from 14 to 70 , with higher scores indicating a higher sense of selfefficacy.

\section{Social skills in nursing interactions with mothers}

Social skills encompass both the verbal and non-verbal interpersonal behaviors used to respond appropriately and effectively in interpersonal situations and the cognitive processes that enable such expression [27]. Fusa et al. developed the Social Skills for Nursing as Novice Nurses scale [28]; for this study, one item was deleted because it was deemed inadequate as a social skill. The reliability and validity of the scale after deleting this item were assessed. The factor structure of the responses of 18 participants was tested to confirm that there were no major changes from the original scale to test the scale's validity. Furthermore, the reliability coefficient (Cronbach's $a=0.85)$ was calculated. The developer's permission was obtained to use the revised version. All processes were carried out with a maternity nursing specialist, and the new scale was used to test social skills (SS) in nursing interactions with mothers. The participants assessed these 24 items on a four-point scale ( 1 = Never used; 4 = Always used), and the total possible scores ranged from 24 to 96 , with higher scores indicating the use of more verbal and non-verbal social skills.

\section{Knowledge and skills necessary for breastfeeding support for LPIs}

To assess participants' knowledge on breastfeeding support, a 20-item questionnaire assessing the Knowledge and Skills (K-S Test) necessary for providing breastfeeding support to LPIs, which consisted of two items on basic knowledge about LPIs and 14 items on knowledge and skills related to breastfeeding support for their mothers was created. The items were given five points for each correct answer, and the total score ranged from 0 to 100 . Higher scores indicated better knowledge and skills.

The same test was administered after a certain period to calculate the correlation coefficient and estimate its reliability [29]. The test was first administered to seven people and then to 11 people after a one-month interval. Kendall's Tau was calculated for analysis, which rendered the results for the first test $b=.689, p=.001$, and for the second $b=.881, p=.001$ and confirmed reliability. It was revised based on the advice of the maternity nursing specialist and neonatal pediatrician.

\section{Data collection}

The data were collected between July and April 2019. The data from immediately before and after the intervention were collected at the convention center, while those one month after the intervention were collected by mail. 


\section{Data analysis}

The descriptive statistics for each variable (i.e., degree, range, mean, standard deviation) were calculated. The Shapiro-Wilk test was used to check the normality of the scores for the scales. The baseline scores for the various tests and the t-test were used to check the uniformity of the two groups. Participant characteristics were tested for each nominal scale by the $\chi^{2}$ test, and the t-test was used for ordinal and interval scales. The SBS, SS, and K-S test scores were used to perform the two-way mixed ANOVA. The program (BSLPI vs. NTS) was considered the inter-group factor, and time (immediately before, after, or one month after the intervention) was considered the intra-group factor. The various factors of the scales for which a main effect was observed were further analyzed in a multiple comparison test, and a simple main effect test was performed on the scales in which interactions were observed between the two factors. $P<0.05$ was considered statistically significant, and SPSS Statistics 22.0 (IBM Corp., Armonk, NY, USA) was used for the analyses.

\section{Results}

No adverse events related to the programs were observed. Homogeneity between the BSLPI and NTS groups was confirmed (Tables 2, 3, 4).

Table 2

Two-group comparison by age and years of experience

\begin{tabular}{|lllllllll|}
\hline \multicolumn{7}{|c|}{ BSLPI $(\mathbf{n}=\mathbf{3 2})$} & NTS $(\mathbf{n}=\mathbf{3 1})$ \\
\hline Item & Mean & SD & Mean & SD & $t$ & $d f$ & $p$ \\
\hline Age & 37.8 & 11.5 & 38.8 & 8.1 & .39 & 61 & .695 \\
Years of experience & 12.2 & 10.1 & 12.9 & 7.4 & .32 & 61 & .751 \\
Years of obstetric ward experience & 10.7 & 9.8 & 10.4 & 6.6 & .11 & 61 & .913 \\
\hline Unpaired t-test. & & & & & & & \\
\hline BSLPI: breastfeeding support for late preterm infants; NTS: non-technical skills \\
\hline
\end{tabular}


Table 3

Inter-group comparison of participants' characteristics

\begin{tabular}{|c|c|c|c|c|c|c|c|c|}
\hline \multirow[b]{2}{*}{ Item } & & \multicolumn{2}{|c|}{$\begin{array}{l}\text { BSLPI }(n= \\
32)\end{array}$} & \multicolumn{2}{|c|}{$\begin{array}{l}\text { NTS }(n= \\
31)\end{array}$} & \multirow[b]{2}{*}{$d f$} & \multirow[b]{2}{*}{$\chi^{2}$} & \multirow[b]{2}{*}{$p$} \\
\hline & & $n$ & $\%$ & $n$ & $\%$ & & & \\
\hline \multirow{2}{*}{$\begin{array}{l}\text { Breastfeeding experience } \\
(\mathrm{Y} / \mathrm{N})\end{array}$} & Y & 15 & 46.9 & 20 & 64.5 & \multirow[t]{2}{*}{1} & \multirow[t]{2}{*}{1.69} & \multirow[t]{2}{*}{.207} \\
\hline & $\mathrm{N}$ & 17 & 53.1 & 11 & 35.5 & & & \\
\hline \multirow[t]{2}{*}{ Nurses midwives ${ }^{a}$ (No.) } & Midwives & 30 & 93.8 & 28 & 90.3 & \multirow[t]{2}{*}{2} & \multirow[t]{2}{*}{1.05} & \multirow[t]{2}{*}{.592} \\
\hline & Nurses & 2 & 6.3 & 2 & 6.5 & & & \\
\hline \multirow[t]{3}{*}{ Facility status } & Hospital & 20 & 62.5 & 18 & 58.1 & \multirow[t]{3}{*}{2} & \multirow[t]{3}{*}{.5 .5} & \multirow[t]{3}{*}{.757} \\
\hline & Clinic & 4 & 12.5 & 6 & 19.4 & & & \\
\hline & $\begin{array}{l}\text { Midwife } \\
\text { center }\end{array}$ & 8 & 25.0 & 7 & 22.6 & & & \\
\hline \multicolumn{9}{|l|}{$\mathrm{X}^{2}$ test } \\
\hline
\end{tabular}

Table 4

Inter-group baseline comparison by each evaluative index

\begin{tabular}{|c|c|c|c|c|c|c|c|}
\hline & \multicolumn{2}{|c|}{$\begin{array}{l}\text { BSLPI } \\
(n=32)\end{array}$} & \multicolumn{5}{|c|}{$\begin{array}{l}\text { NTS } \\
(n=31)\end{array}$} \\
\hline & Mean & $S D$ & Mean & $S D$ & $t$ & $d f$ & $p$ \\
\hline SBS scale scores & 47.8 & 10.3 & 50.5 & 7.6 & 1.2 & 61 & .245 \\
\hline SS scale scores & 74.2 & 11.0 & 74.6 & 8.9 & 0.2 & 61 & .217 \\
\hline K-S test scores & 44.8 & 12.0 & 45.7 & 12.0 & 0.3 & 61 & .926 \\
\hline \multicolumn{8}{|l|}{ Unpaired t-test. } \\
\hline \multicolumn{8}{|c|}{$\begin{array}{l}\text { BSLPI: breastfeeding support for late preterm infants; NTS: non-technical skills; LPIs: late preterm } \\
\text { infants }\end{array}$} \\
\hline \multicolumn{8}{|c|}{ SBS : Self-efficacy of breastfeeding support. } \\
\hline \multicolumn{8}{|c|}{ SS : Social skills in nursing interactions with mothers. } \\
\hline K-S : Knowledge ar & ecessa & rea & $\lg s$ & $\mathrm{~L}$ & & & \\
\hline
\end{tabular}


The ages and years of experience in an obstetrics ward of participants in the BSLPI and NTS groups were $37.8 \pm 11.5$ years and $38.8 \pm 8.1$ years $(t=.39, p=.695)$ and $10.7 \pm 9.8$ years and $10.4 \pm 6.6$ years $(t=.11$, $p=.913)$, respectively. There was no significant difference in the number of nurses and midwives between the two groups $\left(\chi^{2}=1.05, p=.592\right)$. Further, there were no significant differences in baseline scores for the SBS $(t=1.2, p=.245)$, SS $(t=1.0, p=.217)$, and $K-S(t=0.3, p=.926)$ scales.

\section{Outcomes}

Of the 69 nurses and midwives who applied to participate in the study, 36 were randomly assigned to the intervention group and 33 to the control group. Of 36 participants assigned to the intervention group, three did not undergo the intervention due to poor health and one to an unexpected change in work schedule. Of the 33 in the control group, one could not undergo the intervention owing to an unexpected change in work schedule and one to an emergency. The number of participants who could be followed up and their proportions in the intervention and control groups immediately before, immediately after, and one month after the intervention were 32 (89.9\%) and 31 (94.0\%), 32 (83.4\%) and 31 (91.0\%), and 30 (83.4\%) and 30 (91.0\%), respectively. Three who underwent the intervention but could not be followed up $(4.8 \%)$ were treated as missing values. The data from immediately after the intervention were imputed such that 63 participants were ultimately included in the analysis (follow-up rate 87.0\%; Fig. 1).

\section{Effects of the BSLPI intervention}

The mean SBS scale score in the BSLPI group was significantly higher immediately after (mean $=55.7$, standard deviation $(S D)=8.0, p=.001)$ and one month after (mean $=57.3, S D=8.6, p=.001)$ the intervention than immediately before it ( mean $=47.8, S D=10.3$; Table 5, 6; Fig. 2 ). 
Table 5

Evaluation of each index by time and program differences ( $N=63$; BSLPI: $n=32 ; \mathrm{NTS}: \mathrm{n}=31$ )

\begin{tabular}{|c|c|c|c|c|c|c|c|}
\hline & & Source & ss & $D f$ & $M S$ & $F$ & $P$ \\
\hline \multirow[t]{5}{*}{ SBS scale scores } & \multirow[t]{3}{*}{ Inter-group } & Time & 1320.4 & 2 & 660.2 & 24.9 & .001 \\
\hline & & Time $\mathrm{x}$ program & 465.5 & 2 & 232.7 & 8.8 & .001 \\
\hline & & Error & 3230.0 & 122 & 26.4 & & \\
\hline & \multirow[t]{2}{*}{ Intra-group } & Program & 147.1 & 1 & 147.1 & 0.9 & .346 \\
\hline & & Error & 4652.2 & 16 & 290.8 & & \\
\hline \multirow[t]{5}{*}{ SS scale scores } & \multirow[t]{3}{*}{ Inter-group } & Time & 736.6 & 2 & 368.3 & 15.4 & .001 \\
\hline & & Time $\mathrm{x}$ program & 446.5 & 2 & 223.3 & 9.4 & .001 \\
\hline & & Error & 1130.0 & 68 & & & \\
\hline & \multirow[t]{2}{*}{ Intra-group } & Program & 718.8 & 1 & 718.8 & 2.8 & .098 \\
\hline & & Error & 15529.1 & 61 & 254.6 & & \\
\hline \multirow[t]{5}{*}{ K-S test scores } & \multirow[t]{3}{*}{ Inter-group } & Time & 18293.6 & 2 & 9146.8 & 135.9 & .001 \\
\hline & & Time $\mathrm{x}$ program & 11628.5 & 2 & 5814.2 & 86.4 & .001 \\
\hline & & Error & 8214.1 & 122 & 67.3 & & \\
\hline & \multirow[t]{2}{*}{ Intra-group } & Program & 20582.1 & 1 & 20582.1 & 80.6 & .001 \\
\hline & & Error & 4888.5 & 34 & 143.8 & & \\
\hline \multicolumn{8}{|c|}{ Two-way mixed ANOVA for evaluating the difference in mean scores by time and program. } \\
\hline \multicolumn{8}{|c|}{$\begin{array}{l}\text { BSLPI: breastfeeding support for late preterm infants; NTS: non-technical skills; LPIs: late preterm } \\
\text { infants }\end{array}$} \\
\hline \multicolumn{8}{|c|}{ SBS : Self-efficacy of breastfeeding support. } \\
\hline \multicolumn{8}{|c|}{ SS : Social skills in nursing interactions with mothers. } \\
\hline
\end{tabular}


Table 6

Testing of simple main effects to evaluate each index ( $N=63$; BSLPI: $n=32 ;$ NTS: $n=31$ )

\begin{tabular}{|c|c|c|c|c|c|c|c|}
\hline & & Source & SS & $d f$ & $M S$ & $F$ & $p$ \\
\hline \multirow{5}{*}{$\begin{array}{l}\text { SBS scale } \\
\text { scores }\end{array}$} & \multirow{3}{*}{$\begin{array}{l}\text { Program (intra- } \\
\text { group) }\end{array}$} & Pre- & 112.4 & 1 & 112.4 & 1.6 & .213 \\
\hline & & Post- & 269.6 & 1 & 269.6 & 3.7 & .055 \\
\hline & & $\begin{array}{l}1 \text { month } \\
\text { post- }\end{array}$ & 230.7 & 1 & 230.7 & 3.2 & .075 \\
\hline & \multirow[t]{2}{*}{ Time (inter-group) } & BSLPI & 1663.9 & 2 & 832.0 & 11.5 & .001 \\
\hline & & NTS & 146.2 & 2 & 73.1 & 1.0 & .365 \\
\hline \multirow[t]{5}{*}{ SS scale scores } & \multirow{3}{*}{$\begin{array}{l}\text { Program (intra- } \\
\text { group) }\end{array}$} & Pre- & 2.5 & 1 & 2.5 & 0.1 & .876 \\
\hline & & Post- & 470.1 & 1 & 470.1 & 406 & .032 \\
\hline & & $\begin{array}{l}1 \text { month } \\
\text { post- }\end{array}$ & 692.2 & 1 & 692.2 & 6.8 & .010 \\
\hline & \multirow[t]{2}{*}{ Time (inter-group) } & BSLPI & 1178.5 & 2 & 589.3 & 5.8 & .003 \\
\hline & & NTS & 23.0 & 2 & 11.5 & 0.1 & .892 \\
\hline \multirow[t]{5}{*}{ K-S test scores } & \multirow{3}{*}{$\begin{array}{l}\text { Program (intra- } \\
\text { group) }\end{array}$} & Pre- & 10.1 & 1 & 10.1 & 0.8 & .781 \\
\hline & & Post- & 20205.1 & 1 & 20205.1 & 155.4 & .001 \\
\hline & & $\begin{array}{l}1 \text { month } \\
\text { post- }\end{array}$ & 11995.4 & 1 & 11995.4 & 92.3 & .001 \\
\hline & \multirow[t]{2}{*}{ Time (inter-group) } & BSLPI & 29803.6 & 2 & 14901.8 & 114.6 & .001 \\
\hline & & NTS & 582.3 & 2 & 291.1 & 2.2 & .109 \\
\hline \multicolumn{8}{|c|}{$\begin{array}{l}\text { Multiple comparisons were made using the Bonferroni method to evaluate differences in self-efficacy, } \\
\text { social skills, and knowledge by time and program differences. }\end{array}$} \\
\hline \multicolumn{8}{|c|}{$\begin{array}{l}\text { BSLPI: breastfeeding support for late preterm infants; NTS: non-technical skills; LPIs: late preterm } \\
\text { infants }\end{array}$} \\
\hline \multicolumn{8}{|c|}{ SBS : Self-efficacy of breastfeeding support. } \\
\hline \multicolumn{8}{|c|}{ SS : Social skills in nursing interactions with mothers. } \\
\hline
\end{tabular}

The mean SS scale score in the BSLPI group was significantly higher immediately after (mean $=80.5$, SD $=10.9, p=.041)$ and one month after (mean $=82.5, S D=10.1, p=.004)$ the intervention than immediately before it (mean $=74.2, S D=11.0$ ). Moreover, the mean score of the BSLPI group immediately after the intervention ( mean $=80.5, S D=10.9)$ and one month after the intervention (mean $=82.5, S D=10.1$ ) was 
significantly higher $(\mathrm{p}=.032$ and $\mathrm{p}=.010$, respectively) than the corresponding values in the NTS group ( mean $=75.0, \mathrm{SD}=9.7$, and mean $=75.8, \mathrm{SD}=9.4$, respectively; Table 5, 6; Fig. 3).

The mean K-S test scores of the BSLPI group were significantly higher immediately after (mean $=84.5$, $S D=8.3, p=.001$ ) and one month after the intervention (mean $=79.3, S D=11.3, p=.001$ ) than immediately before it (mean $=44.8, S D=12.0)$. Moreover, the mean scores of the BSLPI group immediately after the intervention (mean $=84.5, S D=8.3, p=.001$ ) and one month after the intervention (mean $=79.3, S D=11.3, p=.001$ ) were significantly higher than the corresponding scores in the NTS group $($ mean $=48.7, S D=11.9, p=.001$ and mean $=51.8, S D=12.6, p=.001$, respectively; Tables 5, 6; Fig. 4).

\section{Discussion}

This two-group parallel randomized controlled trial examined an educational program specialized in breastfeeding support for LPIs. Previous programs seeking to educate nurses and midwives on LPI care have conducted single-group assessments [16].

The BSLPI intervention resulted in significantly higher scores on the three parameters immediately after and one month after the intervention compared to immediately before it. SS and K-S test scores were significantly higher in the BSLPI group immediately after and one month after the intervention than in the NTS group.

According to a previous report, the SBS scale score may have increased because watching the video allowed participants to revise their knowledge or techniques [17]. In another report, using the acquired knowledge and skills in a counseling setting with the mother allowed healthcare workers to increase their self-efficacy related to breastfeeding support [30].

There are two possible reasons why the intervention in this study increased the SBS scale scores. First, the gaps between required breastfeeding support for LPIs and actual breastfeeding support offered in individual facilities may have been clarified, together with information sharing between participants and a focus on behaviors aimed at solving the problems. After providing information on interventions deemed effective in breastfeeding support for LPIs, such as starting breastfeeding early, pumping, and continuous skin-to-skin contact or kangaroo mother care [20], the implementation status at the participants' facilities, and opinions on challenges related to their implementation were discussed within groups. This was done based on a previous finding that there are fewer resources and less support for mothers of LPIs to breastfeed successfully than mothers of term infants [10]. Some of the individual participants might have understood effective interventions in breastfeeding support for LPIs, but their organizations as a whole were not implementing them. Participants could draw out the problems they faced in their facilities, investigate for solutions, and discover aspects that could benefit from improvement in their facilities through the BSLPI intervention. 
Second, the simulation learning method was adopted, which could have given participants the confidence to apply the knowledge or skills they acquired. Divided into small groups, participants took turns playing the role of the breastfeeding supporter and the mother. The simulation scenario was set to be similar to actual breastfeeding support situations, consisting of a baby-mother pair, with the following details: three days postpartum, LPI body weight loss of $7.8 \%$, sleepy infant, exhausted mother, and mother only producing a small amount of breastmilk. This scenario was intended to determine if participants could use appropriate knowledge on breastfeeding support for LPIs who were losing weight and aimed to consolidate that knowledge. At the end of the simulation, participants engaged in "reflection" and "discussion" for a debriefing session to promote autonomous thinking and future behavioral changes. In the debriefing session, they experienced other participants validating that they could individually simulate the scenario. Bandura named four sources of information that influence awareness of self-efficacy [31]. In this intervention, participants may have increased their SBS scale scores by applying two sources of knowledge: enactive mastery experience, which consists of the awareness of achievement, and verbal persuasion, which others convinced them that they can achieve their goals.

Although the differences between the programs were not significant, the NTS group also saw an increase in mean scores immediately after and one month after the intervention compared to those before the intervention. It is possible that the control condition also contained elements that increased self-efficacy related to breastfeeding support; therefore, it warrants revisions so that the effects of the BSLPI intervention can be tested against a control that has no effects on the participants assigned to it.

Previous educational programs on breastfeeding for healthcare professionals involved interventions for improving communication. However, few studies assessed the effects of the interventions [32-35]. This study assessed the verbal and non-verbal skills used for maintaining good relationships with mothers and revealed that an intervention could be helpful in this regard, which is explained by two possible reasons. First, the intervention simulated a realistic, commonly encountered clinical situation. The case used in the training was of an exhausted mother of an LPI reluctant to breastfeed. Previous studies have reported that cases used in training should simulate specific scenes that frequently occur in clinical settings to increase the frequency of participants' application of their communication skills [36, 37]. This study supported these results.

Second, reflecting on their tendencies after training allowed participants to apply their social skills in clinical settings. Participants scored significantly higher one month after the intervention than immediately after it, and their frequency of using social skills had increased. During training, participants experienced both the mother and the breastfeeding supporter's roles. This experience may have helped participants improve their communication skills by increased use of the social skills necessary for maintaining good human relationships [27]. This might also increase SS scale scores and could be useful for clinical education for nurses and midwives.

Until now, studies assessing the effectiveness of educational interventions in medical facilities that provide breastfeeding support have reported that such interventions allow medical staff to improve their 
knowledge [34, 38]. However, we were unable to identify any studies where the interventions were specific to breastfeeding support for LPIs and assessed the benefits of such a program. Therefore, we created a test of the knowledge and skills necessary for breastfeeding support based on guidelines developed by experts $[20,39]$. The K-S test scores were significantly higher in the BSLPI group than in the NTS group. The higher scores are attributed to the fact that the content of expert guidelines was summarized and explained, making it easier for participants to comprehend. Breastfeeding support for LPIs is believed to require strategic planning and execution [20]. Therefore, we created a simple chart depicting the types of care needed at various stages, based on references such as the Ten Steps to Successful Breastfeeding [5], The Academy of Breastfeeding Medicine Clinical Protocol\#10 [20], and the Neo-BFHI Core document [40]. Participants used this chart in the group work and took it home after the program, which allowed them to carry forward the acquired knowledge and skills to their workplaces.

The program we developed included clarification of issues at each facility and facilitated sharing among participants; it also helped in the acquisition of knowledge to deal with the relevant issues, made use of exercises to integrate knowledge and skills, and implemented pretend play among the participants. This program is expected to be used to improve the skills of nurses and midwives involved in breastfeeding support for LPIs.

Regarding clinical application, in order to strategically implement breastfeeding support for LPIs as an organization, we hope that nurses and midwives involved in providing breastfeeding support will create a document that summarizes who will provide which intervention and at what stage, as this will foster clarity on the functions to be performed and how they are to be performed. Furthermore, we believe that the quality of breastfeeding support for LPIs can be improved throughout the organization by actually using, evaluating, and revising the created document.

\section{Limitations}

First, the sample size was relatively small. Second, there is a need to examine methods to make the study more interactive for participants. Third, it is necessary to consider which method of delivering the educational program is most effective: face-to-face, online, or a mixed method. Finally, while the quality of the educational program was ensured because the facilitator was a researcher, to disseminate it widely, it is necessary to train facilitators who can ensure its reproducibility.

\section{Conclusions}

The BSLPI program was found to improve participants' self-efficacy for providing breastfeeding support, enhance social skills in nursing interactions with mothers, and increase the knowledge and skills necessary for providing breastfeeding support to mothers of LPIs.

Nurses and midwives who participate in the BSLPI program can acquire skills to apply the interventions deemed particularly important for breastfeeding LPIs in clinical practice. An increase in the number of 
nurses and midwives capable of providing breastfeeding support that is optimal for both LPIs and their mothers is expected to increase the number of breastfed LPIs.

\section{Abbreviations}

LPI: Late preterm infant

BSLPI: Breastfeeding support education for late-term preterm infants

NTS: Non-technical skills

SBS: Self-Efficacy of Breastfeeding Support

SS: Social skills in nursing interactions with mothers

K-S: Knowledge and skills necessary for breastfeeding support for LPIs

\section{Declarations}

\section{Ethics approval and consent to participate}

Approval was obtained from the Research Ethics Review Committee, Japanese Red Cross College of Nursing (2018-060), and the ethics review boards of the facilities targeted for research (201838).

Participants provided written informed consent to participate in the study. This trial was retrospectively registered with the University Hospital Medical Information Network (UMIN; R000040145UMIN000035227).

\section{Consent for publication}

Not applicable.

\section{Availability of data and materials}

Not applicable.

\section{Competing interests}

The authors declare that they have no competing interests.

\section{Funding}

This research was supported by JSPS KAKENHI Grant Number JP17k12302.

\section{Authors' contributions}


IS conceived the study, designed it, collected the data, analyzed and interpreted it, and wrote the paper. MI was involved in project conception, data analysis, interpretation, and drafting of the manuscript. Both the authors read and approved the final manuscript.

\section{Acknowledgments}

We thank I. Sasaki, K. Abe, R. Emoto, and T. Tanaka for lending their expertise to the development and implementation of the educational programs. Finally, we would like to thank K. Endo for his advice on interpreting the results of the data analysis.

\section{References}

1. Blencowe H, Cousens S, Chou D, Oestergaard M, Say L, Moller A, et al. Born too soon: the global epidemiology of 15 million preterm births. Reprod Health. 2013;10(Suppl 1):S2.

2. Gianni ML, Consonni D, Liotto N, Roggero P, Morlacchi L, Piemontese P, et al. Does human milk modulate body composition in late preterm infants at term-corrected age? Nutrients. 2016;8(10):664.

3. Forsgren M, Isolauri E, Salminen S, Rautava S. Late preterm birth has direct and indirect effects on infant gut microbiota development during the first six months of life. Acta Paediatr. 2017;106(7):1103-9.

4. Belfort MB, Gillman MW, Buka SL, Casey PH, McCormick MC. Preterm infant linear growth and adiposity gain: trade-offs for later weight status and intelligence quotient. J Pediatr. 2013;163(6):1564-9.e2.

5. World Health Organization. Protecting, promoting, and supporting breastfeeding in facilities providing maternity and newborn services: the revised Baby-Friendly Hospital Initiative 2018 implementation guidance. 2018. https://www.who.int/nutrition/publications/infantfeeding/bfhiimplementation/en/. Accessed 4 Mar 2021.

6. Eidelman Al. The challenge of breastfeeding the late preterm and the early-term infant. Breastfeed Med. 2016;11:99.

7. Philipp BL. ABM Clinical Protocol \#7: model breastfeeding policy (revision 2010). Breastfeed Med. 2010;5(4):173-7.

8. Asadi S, Bloomfield FH, Harding JE. Nutrition in late preterm infants. Semin Perinatol. 2019;43(7):151160.

9. Rayfield S, Oakley L, Quigley MA. Association between breastfeeding support and breastfeeding rates in the UK: a comparison of late preterm and term infants. BMJ Open. 2015;5(11):e0091442015-009144.

10. Goyal NK, Attanasio LB, Kozhimannil KB. Hospital care and early breastfeeding outcomes among late preterm, early-term, and term infants. Birth. 2014;41(4):330-8.

11. Hackman NM, Alligood-Percoco N, Martin A, Zhu J, Kjerulff KH. Reduced breastfeeding rates in firstborn late preterm and early term infants. Breastfeed Med. 2016;11(3):119-25. 
12. Dosani A, Hemraj J, Premji SS, Currie G, Reilly SM, Lodha AK, et al. Breastfeeding the late preterm infant: experiences of mothers and perceptions of public health nurses. Int Breastfeed J. 2017;12:23017-0114-0.

13. Cescutti-Butler L, Hemingway A, Hewitt-Taylor J. "His tummy's only tiny" - scientific feeding advice versus women's knowledge. Women's experiences of feeding their late preterm babies. Midwifery. 2019;69:102-9.

14. Alexander T, Bloomfield FH. Nutritional management of moderate-late preterm infants: survey of current practice. J Paediatr Child Health. 2019;55(3):338-42.

15. Allistair Jensen. Late preterm babies - their problems and care. 2011.

https://www.infantjournal.co.uk/pdf/inf_040_ret.pdf. Accessed 4 Mar 2021.

16. Cooper AL, Brown JA, O’Connor T, Eccles S. Improving the clinical skills and knowledge of midwives and nurses caring for late preterm neonates. J Contin Educ Nurs. 2019;50(12):551-6.

17. Wallace LM, Ma Y, Qiu LQ, Dunn OM. Educational videos for practitioners attending baby friendly hospital initiative workshops supporting breastfeeding positioning, attachment and hand expression skills: effects on knowledge and confidence. Nurse Educ Pract. 2018;31:7-13.

18. Nagashima K. A computerized number generator through the stratified block randomization. 2017. https://nshi.jp/contents/js/randblock/. Accessed 5 Mar 2021.

19. Faul F, Erdfelder E, Lang A, Buchner A. G*Power 3: a flexible statistical power analysis program for the social, behavioral, and biomedical sciences. Behav Res Methods. 2007; 39(2):175-91.

20. Boies EG, Vaucher YE. ABM Clinical Protocol \#10: breastfeeding the late preterm (34-36 6/7 weeks of gestation) and early term infants (37-38 6/7 weeks of gestation), second revision 2016. Breastfeed Med. 2016;11:494-500.

21. Shields A, Flin R. Paramedics' non-technical skills: a literature review. Emerg Med J. 2013;30(5):3504.

22. Helmreich RL. Managing human error in aviation. Sci Am. 1997;276(5):62-7.

23. Langdalen H, Abrahamsen EB, Sollid SJM, Sorskar LIK, Abrahamsen HB. A comparative study on the frequency of simulation-based training and assessment of non-technical skills in the Norwegian ground ambulance services and helicopter emergency medical services. BMC Health Serv Res. 2018;18(1):509-018-3325-1.

24. Fletcher G, Flin R, McGeorge P, Glavin R, Maran N, Patey R. Anaesthetists' non-technical skills (ANTS): evaluation of a behavioural marker system. Br J Anaesth. 2003;90(5):580-8.

25. Pires SMP, Monteiro SOM, Pereira AMS, Stocker JNM, Chaló DM, Melo EMOP. Non-technical skills assessment scale in nursing: construction, development and validation. Rev Lat Am Enfermagem. 2018;26:e3042.

26. Toyama N, Kurihara K, Muranaka M, Kamibeppu K. Factors influencing self-efficacy in breastfeeding support among public health nurses in Japan. 2013. https://www.scirp.org/journal/PaperInformation.aspx?PaperID=40906. Accessed 4 May 2021. 
27. Aikawa A, Tumura T. Social skills and interpersonal relationships: helping self-expression Tokyo: SEISHIN SHOBO; 1996.

28. Fusa M, Mayumi M, Mutsumi C. Social skills for nursing as novice nurses. J Facult Nurs, Iwate Prefectural University. 2002;4:25-35.

29. Okada O. Reliability in psychology and psychological measurement, with focus on Cronbach's alpha. Ann Rep Edu Psy Jap. 2015;54:71-83.

30. Kronborg H, Vaeth M, Olsen J, Harder I. Health visitors and breastfeeding support: influence of knowledge and self-efficacy. Eur J Public Health. 2008;18(3):283-8.

31. Bandura A. Social learning theory. 1st ed. Tokyo: Kaneko Shobo; 2012.

32. Rea MF, Venancio SI, Martines JC, Savage F. Counselling on breastfeeding: assessing knowledge and skills. Bull World Health Organ. 1999;77(6):492-8.

33. Tongun JB, Tumwine JK, Ndeezi G, Sebit MB, Mukunya D, Nankunda J, et al. The effect of health worker training on early initiation of breastfeeding in South Sudan: a hospital-based before and after study. Int J Environ Res Public Health. 2019;16(20):3917.

34. Balogun 0O, Dagvadorj A, Yourkavitch J, Lopes KdS, Suto M, Takemoto Y, et al. Health facility staff training for improving breastfeeding outcome: a systematic review for step 2 of the baby-friendly hospital initiative. Breastfeed Med. 2017;12(9):537-46.

35. Colaceci S, Giusti A, Chapin EM, Bettinelli ME, De Angelis A, Zambri F, et al. E-learning to improve healthcare professionals' attitudes and practices on breastfeeding. Breastfeed Med. 2017;12(10):629-36.

36. Brown CE, Back AL, Ford DW, Kross EK, Downey L, Shannon SE, et al. Self-assessment scores improve after simulation-based palliative care communication skill workshops. Am J Hosp Palliat Care. 2018;35(1):45-51.

37. Newey CR, Bell R, Burks M, Nattanmai P. A new strategy in neurocritical care nurse continuing stroke education: a hybrid simulation pilot study. Electron Physician. 2017;9(5):4255-60.

38. Watkins AL, Dodgson JE. Breastfeeding educational interventions for health professionals: a synthesis of intervention studies. J Spec Pediatr Nurs 2010;15(3):223-32.

39. Briere CE, Lucas R, McGrath JM, Lussier M, Brownell E. Establishing breastfeeding with the late preterm infant in the NICU. J Obstet Gynecol Neonatal Nurs. 2015;44(1):E1-2.

40. Nordic and Quebec Working Group Members. Neo-BFHI package. 2015. https://ilca.org/neo-bfhi/. Accessed 4 Mar 2021.

\section{Figures}


Incorporation

Allocation

Assigned to BSLPla group

$$
(n=36)
$$

Accepting intervention on assignment $(n=32)$

Not accepting intervention on assignment $(n=4)$

(Reasons=physical unwellness, $n=3$ )
Excluded $(n=0)$

Not included in selection criteria $(n=0)$

Withdrawal from participation $(n=0)$

Other reasons $(n=0)$

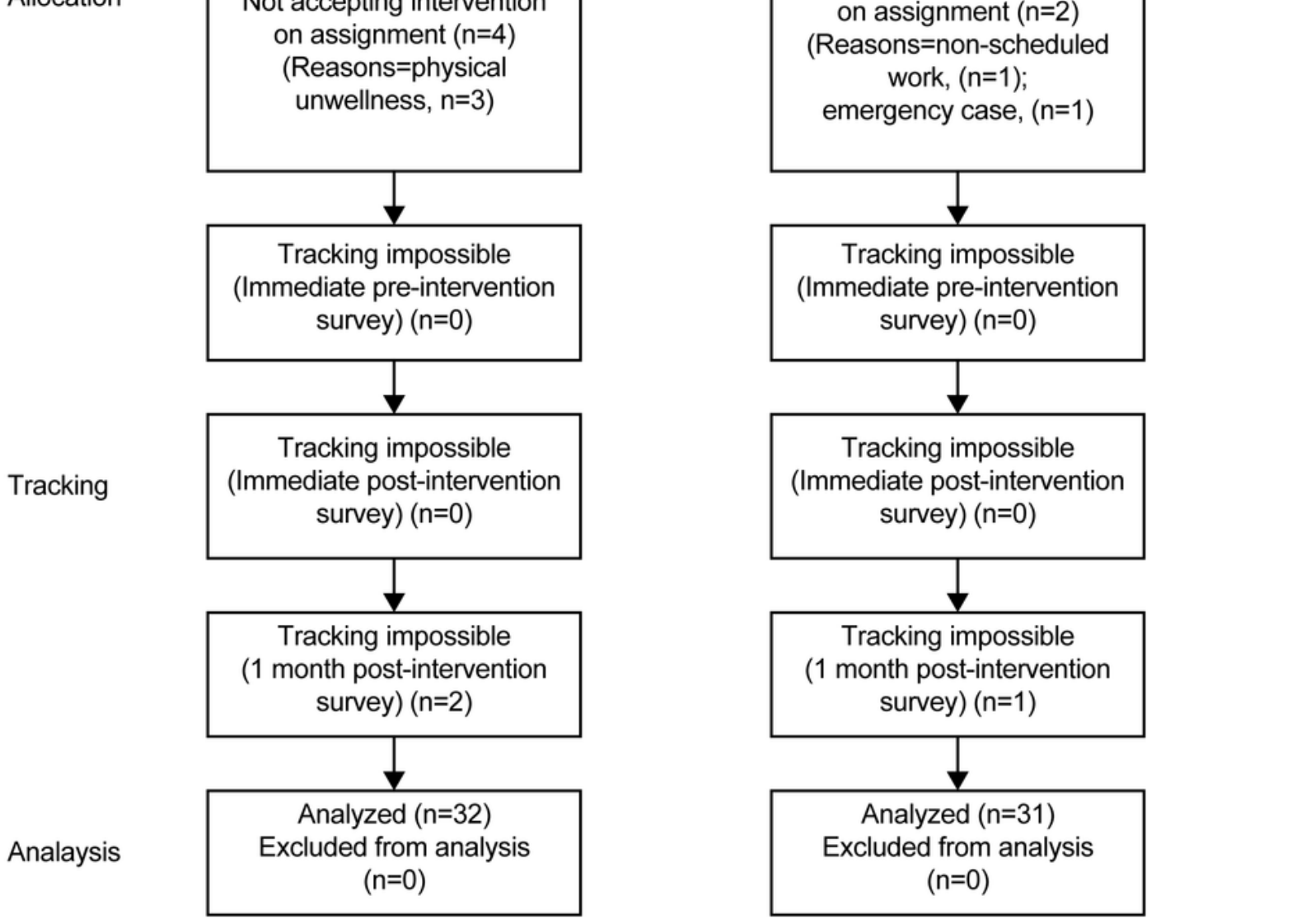

\section{Figure 1}

Participant selection flowchart. BSLPI: breastfeeding support for late preterm infants; NTS: non-technical skills 


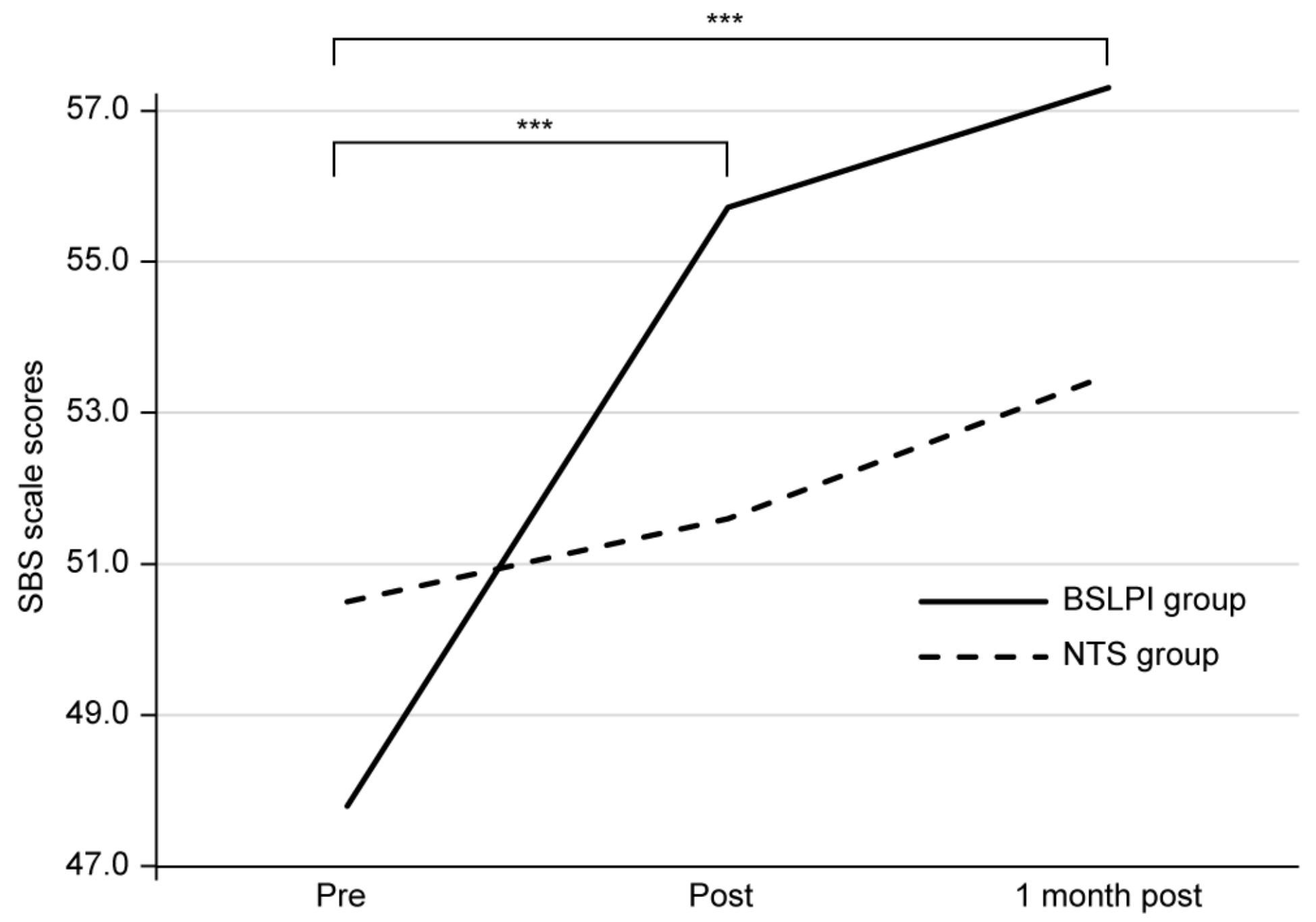

Figure 2

Changes in scores for Self-Efficacy of Breastfeeding Support scale. Higher scores indicate better breastfeeding support self-efficacy. ${ }^{* \star} \mathrm{p}<.001 \mathrm{BSLPI}$ : breastfeeding support for late preterm infants; NTS: non-technical skills SBS : Self-Efficacy of breastfeeding support. 


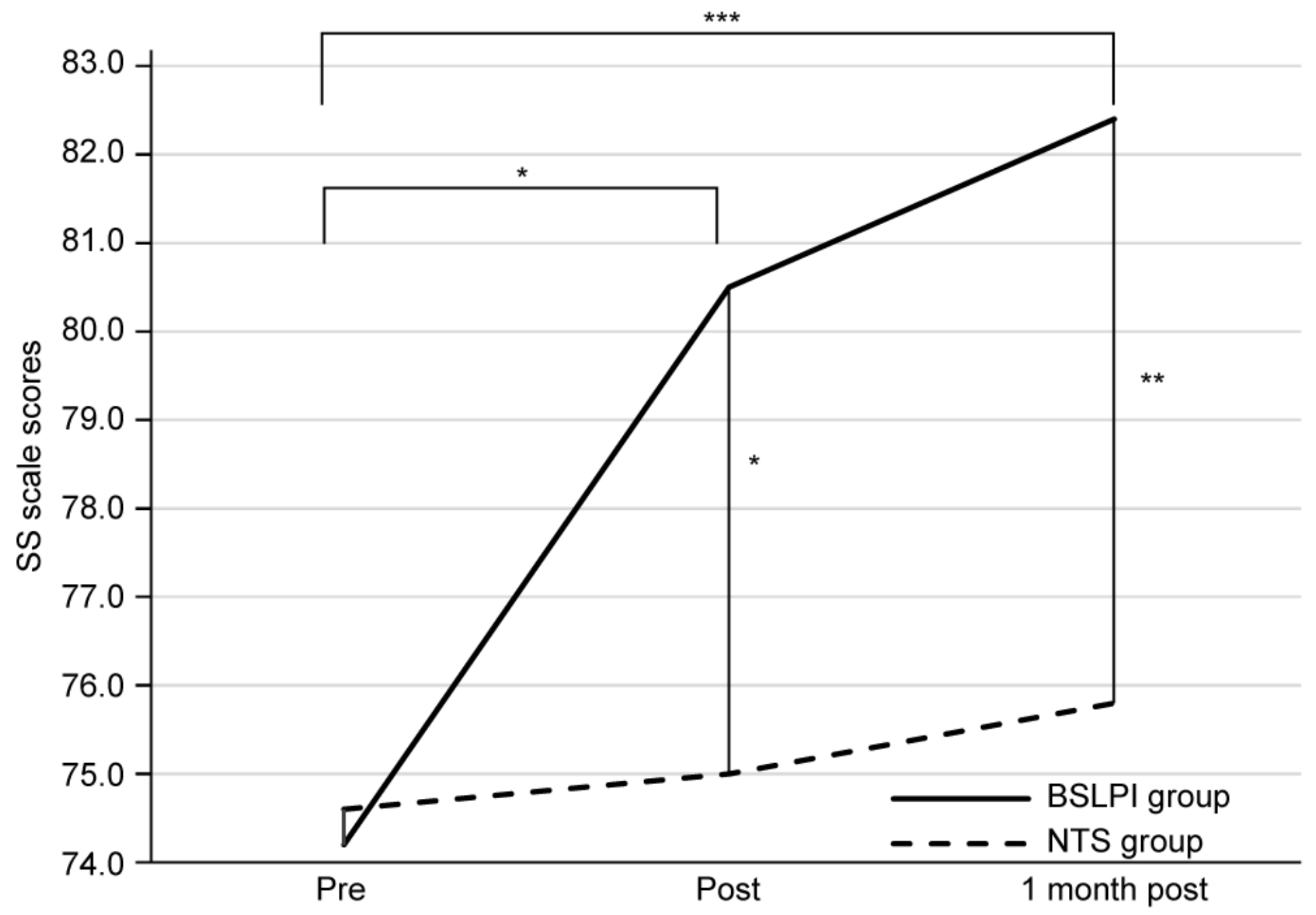

Figure 3

Changes in nurses' and midwives' social skills scores. Higher scores indicate better nursing social skills. ${ }^{*} \mathrm{p} \otimes .05 .{ }^{* \star} \mathrm{p}<.01 .{ }^{* \star *} \mathrm{p}<.001 \mathrm{BSLPI}$ : breastfeeding support late preterm infants; NTS: non-technical skills SS : Social skills in nursing interactions with mothers. 


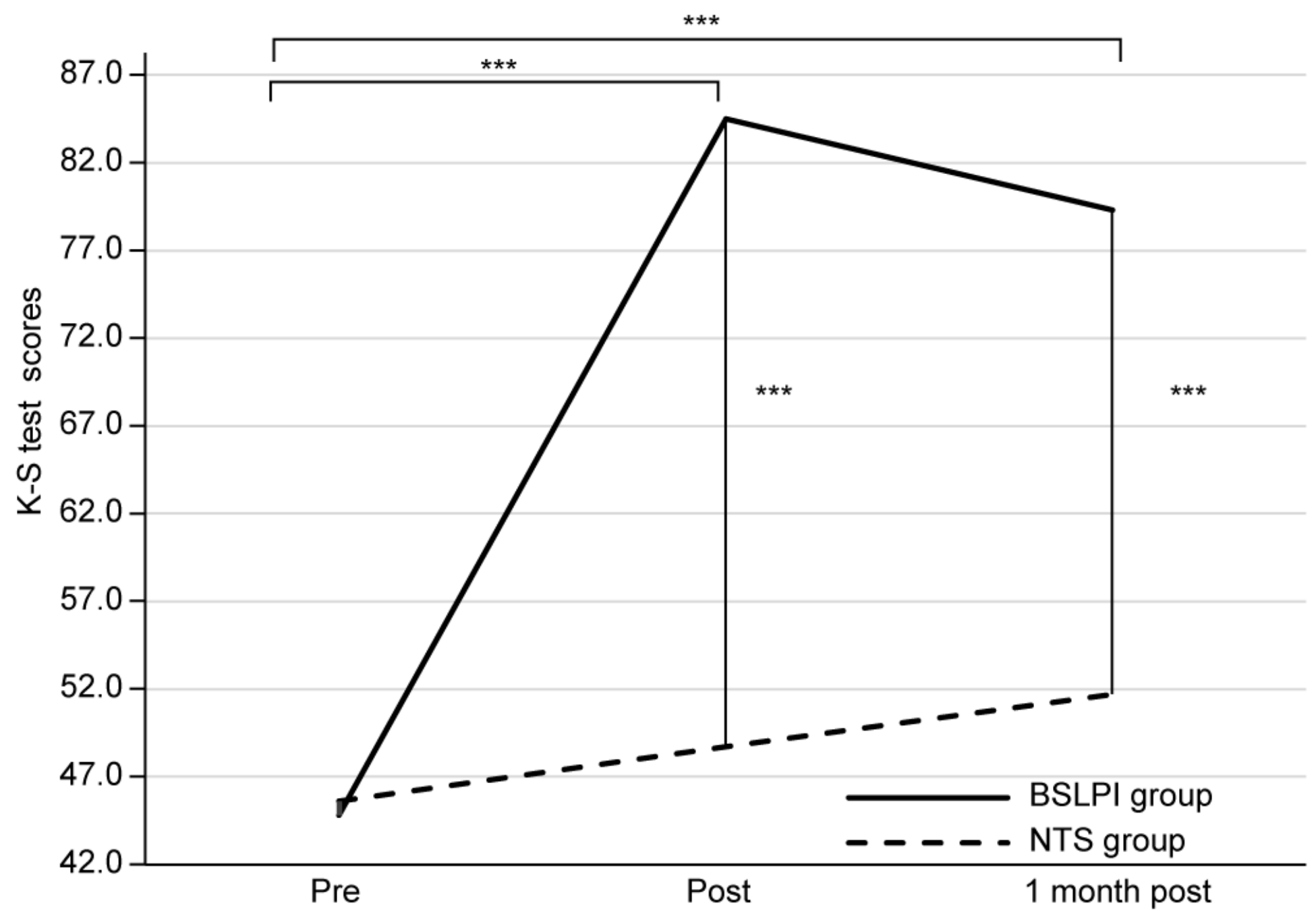

Figure 4

Changes in nurses' and midwives' knowledge and skills scores. Higher scores indicate better knowledge and techniques concerning breastfeeding support for late preterm infants. ${ }^{\star \star \star} p<.001 \mathrm{BSLPI}$ : breastfeeding support for late preterm infants; NTS: non-technical skills K-S : knowledge and skills necessary for breastfeeding support for LPIs. 